\title{
HMGB1 Receptors as Potential Novel Targets for Neuroblastoma
}

\author{
Christian Garcia ${ }^{1}$, Jared Smith², Peter Malicky³, Fletcher White ${ }^{2,3}$ \\ ${ }^{1}$ Indiana Medical Student Program for Research and Scholarship, ${ }^{2}$ Medical \\ Neuroscience Program, ${ }^{3}$ Department of Anesthesia, Indiana University School of \\ Medicine.
}

\begin{abstract}
Background and Hypothesis: The pathogenesis of neuroblastoma remains elusive. In order to further understand the pathogenesis, it is necessary to identify molecular and cellular mechanisms of the cancer. One protein of interest is high-mobility group box 1 protein (HMGB1). HMGB1 has been implicated in cancer including tumor growth, invasion, angiogenesis, metastasis, relapse and therapeutic resistance. HMGB1 isoforms signals via different receptors including the chemokine receptor, CXCR4, receptor for advanced glycation end products (RAGE), and the prominent inflammation-associated receptor, toll-like receptor 4 (TLR4). To gain insight into the possible impact of HMGB1 on the SH-SY5Y neuroblastoma cell line, we determined the degree to which CXCR4, TLR4 and RAGE are present on SH-SY5Y cells. We hypothesize that the presence of these receptors in SH-SY5Y cells may mediate proliferation of neuroblastoma cells and other types of cancer.
\end{abstract}

\section{Experimental Design or Project Methods:}

The SH-SY5Y cell line (ATCC $®$ CRL-2266 ${ }^{\mathrm{TM}}$ ) was derived from a metastatic bone tumor in a 4 year old female. Cells were lysed in lysis buffer, electrophoresed on a $10 \%$ SDS-PAGE, and blotted onto PVDF membrane. After blocking, the membranes were incubated with primary antibodies against the receptor protein overnight at $4{ }^{\circ} \mathrm{C}$, and then with HRP-conjugated secondary antibodies for $1 \mathrm{~h}$. Protein bands were visualized with a SuperSignal West Pico Chemiluminescent Substrate.

\section{Results: To be finalized}

Conclusion and Potential Impact: Investigating SH-SY5Y cell line for the presence of these inflammation-associated receptors could potentially serve as a model to better understand the role of these receptors in cancer research. 